BNL-77344-2007-CP

\title{
Electron Cooling in the Presence of Undulator Fields
}

\author{
A.V. Fedotov, I. Ben-Zvi, D. Kayran, V.N. Litvinenko, \\ E. Pozdeyev, BNL, USA \\ G. Bell, D.L. Bruhwiler, A. Sobol, Tech-X Corp. USA \\ A. Sidorin, A. Smirnov, JINR, Russia
}

Presented at the $22^{\text {nd }}$ Particle Accelerator Conference

Albuquerque, NM

June 25-29, 2007

June 2007

\author{
Collider-Accelerator Department \\ Brookhaven National Laboratory \\ P.O. Box 5000 \\ Upton, NY 11973-5000 \\ wuw.bnl.gov
}

\begin{abstract}
Notice: This manuscript has been authored by employees of Brookhaven Science Associates, LLC under Contract No. DE-AC02-98CH10886 with the U.S. Department of Energy. The publisher by accepting the manuscript for publication acknowledges that the United States Government retains a non-exclusive, paid-up, irrevocable, world-wide license to publish or reproduce the published form of this manuscript, or allow others to do so, for United States Government purposes.
\end{abstract}

This preprint is intended for publication in a journal or proceedings. Since changes may be made before publication, it may not be cited or reproduced without the author's permission. 


\section{DISCLAIMER}

This report was prepared as an account of work sponsored by an agency of the United States Government. Neither the United States Government nor any agency thereof, nor any of their employees, nor any of their contractors, subcontractors, or their employees, makes any warranty, express or implied, or assumes any legal liability or responsibility for the accuracy, completeness, or any third party's use or the results of such use of any information, apparatus, product, or process disclosed, or represents that its use would not infringe privately owned rights. Reference herein to any specific commercial product, process, or service by trade name, trademark, manufacturer, or otherwise, does not necessarily constitute or imply its endorsement, recommendation, or favoring by the United States Government or any agency thereof or its contractors or subcontractors. The views and opinions of authors expressed herein do not necessarily state or reflect those of the United States Government or any agency thereof. 


\title{
ELECTRON COOLING IN THE PRESENCE OF UNDULATOR FIELDS*
}

\author{
A. V. Fedoto"\#, I. Ben-Zvi, D. Kayran, V. N. Litvinenko, E. Pozdeyev, BNL, Upton, NY 11973 \\ G. Bell, D. L. Bruhwiler, A. Sobol, Tech-X Corp., CO, USA \\ A. Sidorin, A. Smirnov, JINR, Dubna, Russia
}

\begin{abstract}
The design of the higher-energy cooler for Relativistic Heavy Ion Collider (RHIC) recently adopted a nonmagnetized approach which requires a low temperature electron beam [1]. However, to avoid significant loss of heavy ions due to recombination with electrons in the cooling section, the temperature of the electron beam should be high. These two contradictory requirements are satisfied in the design of the RHIC cooler with the help of the undulator fields. The model of the friction force in the presence of an undulator field was benchmarked vs direct numerical simulations with an excellent agreement. Here, we discuss cooling dynamics simulations with a helical undulator, including recombination suppression and resulting luminosities.
\end{abstract}

\section{ELECTRON COOLING FOR RHIC-II}

The present performance of the RHIC collider with heavy ions is limited by the process of Intra-Beam Scattering (IBS) [2]. To achieve required luminosities for the future upgrade [3] of the RHIC complex (known as RHIC-II) an electron cooling system was proposed [4]

The baseline of the heavy-ion program for RHIC-II is operation with $\mathrm{Au}$ ions at total energy per beam of 100 $\mathrm{GeV} /$ nucleon. For such an operation, the electron cooling should compensate IBS and provide an increase by about factor of 10 in an average luminosity per store.

For RHIC-II operation with the polarized protons, the electron cooling should assist in obtaining required initial transverse and longitudinal emittances or prevent their significant increase due to IBS. Although IBS is not as severe for protons as for heavy ions, a proposed increase in proton intensity for RHIC-II upgrade makes IBS an important effect as well.

In a traditional low-energy electron cooler, where the magnetic field is used for the transport of an electron beam through the cooling section from the gun to the collector, the longitudinal magnetic field changes the collision kinetics significantly. The magnetic field limits transverse motion of the electrons. As a result, the efficiency of electron cooling is determined mainly by the longitudinal velocity spread of the electrons. Such cooling is typically referred to as "magnetized cooling" $[5,6]$.

However, if an rms velocity spread within electron beam is comparable to the one of the ion beam, the cooling can be done without the help of the strong external magnetic field.

*Work supported by the U.S. DOE Office of Science, Office of Nuclear Physics, including grant \#DE-FG02-04ER84094.

"fedotov@bobl.gov
Such type of cooling is referred to as the "nonmagnetized cooling"; although a weak external field can be still employed, for example, to ensure focusing and alignment of electron and ion beams. The first cooling system which is based on the non-magnetized approach was constructed at the FNAL Recycler ring [7].

Although extensive studies of the magnetized cooling approach for RHIC showed that such approach is feasible [1] and would provide required luminosities for the RHIC-II, the baseline was recently changed to the nonmagnetized one. Electron cooling at RHIC using the nonmagnetized electron beam significantly simplifies the cooler design. The generation and acceleration of the electron bunch without longitudinal magnetic field allows us to reach a low value of the emittance for the electron beam in the cooling section. The cooling rate required for suppression of the Intra-Beam Scattering (IBS) can be achieved with a relatively small charge of the electron bunch $\sim 5 \mathrm{nC}$.

Since non-magnetized cooling requires low temperature of the electrons, a possible problem which one can encounter in cooling of heavy ions is a high recombination rate of ions with the electrons. In the present design, suppression of the ion recombination is based on employing fields of a helical undulator in the cooling section [8]. In the presence of undulator field, electron trajectories have coherent azimuthal angle which helps to suppress recombination. The critical point is to understand how electron cooling force is affected as a result of such recombination suppression, which is explained in this article.

\section{FRICTION FORCE IN THE PRESENCE OF UNDULATOR FIELDS}

In the presence of an undulator field, the trajectories of all the electrons have the same coherent azimuthal angle $\theta$, determined by the undulator period $\lambda$ and magnetic field value $B$ at the axis:

$\theta=\frac{e B \lambda}{2 \pi p c}$,

where $p$ is the electron momentum. Since the recombination cross section is approximately inversely proportional to the electron energy in the ion rest frame, the ion beam life time can be sufficiently improved. One can expect that at impact parameters significantly larger than the electron rotation radius

$r_{0}=\frac{\theta \lambda}{2 \pi}=\frac{e B \lambda^{2}}{4 \pi^{2} p c}$ 
kinematics of the binary collisions will be similar to Rutherford scattering of a free electron. In this case the friction force acting on the ion inside the electron beam with the velocity distribution function $f\left(v_{e}\right)$ can be still calculated with the usual formula:

$$
\vec{F}=-\frac{4 \pi n_{e} e^{4} Z^{2}}{m_{e}} \int L_{c} \frac{\vec{V}_{i}-\vec{v}_{e}}{\left|\vec{V}_{i}-\vec{v}_{e}\right|^{3}} f\left(v_{e}\right) d^{3} v_{e},
$$

where $n_{e}$ is electron density in the Particle Rest Frame (PRF), $v_{e}, V_{i}$ are the electron and ion velocity, $L_{c}-$ Coulomb logarithm:

$L_{c}=\ln \frac{\rho_{\max }}{\rho_{\min }}$.

For the RHIC parameters, the maximum impact parameter is determined by the time of flight of the ion through the cooling section and it is not affected by the undulator field. However, the minimum impact parameter $\rho_{\min }$ which is determined by a relative velocity between an ion and electron as

$\rho_{\min }=\frac{Z e^{2}}{m_{e}} \frac{1}{\left|\vec{V}_{i}-\vec{v}_{e}\right|^{2}}$,

has to be replaced by $r_{0}$ value, in the presence of the undulator field. Therefore, the friction force is expected to be reduced by a factor of the order of $\ln \frac{\rho_{\max }}{\rho_{\min }} / \ln \frac{\rho_{\max }}{r_{0}}$

To make sure that such a representation of the friction force is accurate, an undulator field was implemented in the VORPAL code [9], and numerical simulations were performed for different strength of the magnetic field $B$ and pitch period $\lambda[10]$.

An example of such a comparison between VORPAL simulation (dots with error bars) of the friction force experienced by $\mathrm{Au}$ ion with $\mathrm{Z}=79$ during a single interaction with the electron beam and numerical integration of the force expression in Eq. (3) with the minimum impact parameter calculated according to Eq. (2) is shown in Fig. 1. Simulation parameters were (in the PRF): interaction time $\tau=0.9 \mathrm{nsec}$, for ion velocity of $3.0 \cdot 10^{5}[\mathrm{~m} / \mathrm{s}]$; electrons parameters: $\mathrm{rms}$ velocity spread $\Delta_{\mathrm{I}}=3.0 \cdot 10^{5}[\mathrm{~m} / \mathrm{s}]$ and $\Delta_{\mathrm{l}}=3.0 \cdot 10^{5}[\mathrm{~m} / \mathrm{s}]$ transverse and longitudinal, respectively, density $\mathrm{n}_{\mathrm{e}}=7.32 \cdot 10^{13} \mathrm{~m}^{-3}$. The force $[\mathrm{eV} / \mathrm{m}]$ is plotted vs angle [rad] of an ion velocity vector with respect to the longitudinal direction.

In all simulated cases, it was found that the friction force scales as predicted by a modified logarithm in Eq. (6). This confirmed our expectations that with a modest reduction of the friction force values one can introduce relatively large azimuthal coherent velocity of electrons to suppress recombination.

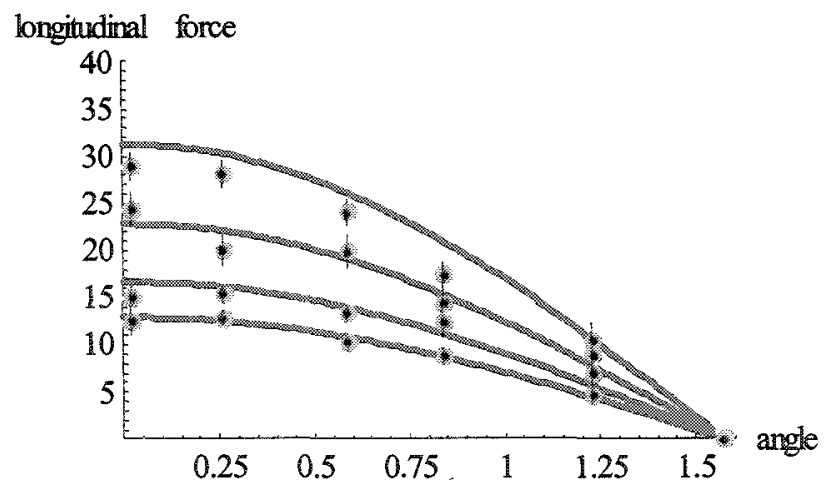

Figure 1: Longitudinal component of the friction force $[\mathrm{eV} / \mathrm{m}]$ vs angle [rad] for the ion with velocity of $3 \times 10^{5}$ $\mathrm{m} / \mathrm{s}$ in PRF. Comparison is shown for zero magnetic field $\mathrm{B}=0$ (upper curve) and for an undulator with $\mathrm{B}=10 \mathrm{G}$ and different periods $\lambda=8,16$ and $24 \mathrm{~cm}$ (lower curve). VORPAL results - dots with error bars; numerical integration - solid lines.

Impact of undulator fields was further investigated by including errors in the alignment of individual sections of the undulator. Even with relatively high offsets of $3 \mathrm{~mm}$ no significant effect on the friction was observed [10]. A detailed study of the effects of magnetic field errors on the friction force was also performed [11].

\section{RECOMBINATION CALCULATION}

The ion beam life time due to recombination in the cooling section is calculated via recombination coefficient $\alpha_{r}$ by the following formula:

$$
\frac{1}{N} \frac{d N}{d t}=-\frac{\alpha_{r} n_{e}}{\gamma^{2}} \frac{l_{c o o l}}{C}
$$

where $\mathrm{C}$ is the ring circumference and $\mathrm{l}_{\text {cool }}$ is the length of the cooler. The recombination coefficient $\alpha_{\mathrm{T}}$ is calculated in PRF by averaging of the classical cross section of radiative recombination (cooling is applied to fully stripped ions) over the distribution function, taking into account coherent velocity of electrons in the undulator.

Radiative recombination of ions in rings with electron coolers was extensively studied experimentally. Perfect agreement between measurements and theoretical prediction for the recombination coefficient was found in a wide range of relative energies between the electrons and ions $(>10 \mathrm{meV})$. However, in the region of extremely small relative energies (which is the region typically used for electron cooling), the measured recombination coefficient for experiments with bare ions was found significantly higher than predicted by standard theory of radiative recombination. In the latest theoretical model [12], which reproduces the measurements, the presence of strong solenoidal magnetic field in the cooling section is important for an additional recombination channel. This mechanism is not expected to occur in the absence of the solenoidal magnetic field as in the RHIC approach based on the non-magnetized cooling. In addition, presence of 
an undulator field introduces effective detuning towards relative energies where agreement between standard theory and measurements is good [13].

Since present design of the cooling section employs large beta-functions ( 400 meters for ions and 500 meters for electrons), the density of electron bunch was reduced compared to initial estimates, which in turn reduced the recombination rate. The parameters of undulator were originally set for magnetic field of $10 \mathrm{G}$ and a period of 8 $\mathrm{cm}$, corresponding to an effective temperature of $30 \mathrm{eV}$ and recombination lifetime of 166 hours. However, recent studies of the optimum point between recombination loss and friction force reduction due to undulator fields suggest that strong suppression of recombination rate is perhaps unnecessary, and that parameters of the undulator may be relaxed to just $2 \mathrm{G}$ with a period of $12 \mathrm{~cm}$.

Table 1. Parameters of electron cooler for RHIC-II.

\begin{tabular}{|l|l|l|}
\hline & Unit & Value \\
\hline kinetic energy & $\mathrm{MeV}$ & 54.3 \\
\hline rf frequency & $\mathrm{MHz}$ & 703.75 \\
\hline bunch frequency & $\mathrm{MHz}$ & 9.38 \\
\hline bunch charge & $\mathrm{nC}$ & 5 \\
\hline $\begin{array}{l}\text { rms emittance, } \\
\text { normalized }\end{array}$ & $\mu \mathrm{m}$ & $<4$ \\
\hline rms momentum spread & & $3 \times 10^{-4}$ \\
\hline rms bunch length & $\mathrm{cm}$ & 0.8 \\
\hline $\begin{array}{l}\text { rms beam radius in cooling } \\
\text { section }\end{array}$ & $\mathrm{cm}$ & 0.4 \\
\hline cooling section length & $\mathrm{m}$ & 100 \\
\hline
\end{tabular}

\section{COOLING PERFORMANCE}

To ensure good cooling performance a quality of the electron beam should not suffer significantly as a result of the electron beam transport in ERL, merging of the electron and ion beam, transport through the cooling section and interactions with the ion beam [1].

With the non-magnetized cooling approach, electron angles in the cooling section should be comparable to the angular spread of the ion beam being cooled. With ion beam $95 \%$ normalized emittance of 15 [mm mrad] and beta-function in the cooling section of 400 [meters], the $\mathrm{rms}$ angular spread of ion beam is $7.6[\mu \mathrm{rad}]$.

In the baseline cooling simulations with $5 \mathrm{nC}$ electron beam we assumed "effective" rms angular spread of the electrons of 8.6 [ $\mu \mathrm{rad}]$, which, for example, corresponds to the electron beam rms normalized emittance (thermal contribution) of $4[\mu \mathrm{m}]$, if no other contributions to electron angular spread are present. An emittance of 3 [ $\mu \mathrm{m}]$ (demonstrated in simulations [1]) corresponds to rms angular spread of 7.5 [ $\mu \mathrm{rad}]$ and allows to accommodate additional contributions from other sources. To have a minimum impact on cooling performance, the goal is to constrain total contribution to the rms angular spread of the electrons to about $10[\mu \mathrm{rad}]$.

Cooling dynamics studies for RHIC-II are being performed using the BETACOOL code [14]. Typical simulation which include electron cooling, IBS, particle loss in collisions (burn-off), loss from the if bucket and recombination is shown in Fig. 2. For details see "RHICII Feasibility Study" document in Ref. [1].

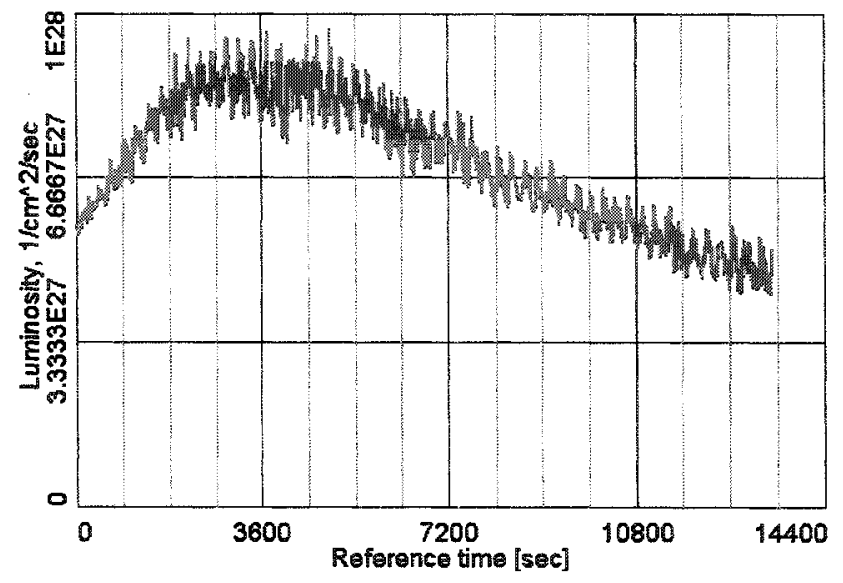

Figure 2 Electron cooling simulation of $\mathrm{Au}-\mathrm{Au}$ luminosity: ion bunch intensity $1 \times 10^{9}, 111$ bunches, $\beta^{*}=0.5 \mathrm{~m}$; using electron bunch parameters from Table 1 . Average luminosity in 4 hour store is $7 \times 10^{27} \mathrm{~cm}^{-2} \mathrm{~s}^{-1}$.

\section{ACKNOWLEDGMENTS}

We would like to thank A. Burov, Ya. Derbenev, W. Fischer, A. Jain, J. Kewisch, T. Roser and the Accelerator Physics and Electron Cooling Groups of RHIC for useful discussions. We are grateful to the VORPAL team of Tech-X Corp. and BETACOOL team of JINR, Russia.

\section{REFERENCES}

[1] Electron cooling for RHIC: http://www.bnl.gov/cad/ecooling

[2] A. Fedotov et al, Workshop HB2006 (KEK,Tsukuba, Japan), pub. by JACoW, p.259, 2006.

[3] A. Fedotov, "RHIC plans towards higher luminosity", these proceedings.

[4] V. Parkhomchuk and I. Ben-Zvi, BNL C-AD Technical Report C-AD/AP/47 (2001).

[5] Ya. Derbenev, A. Skrinsky, Part. Acc. 8, p. 235 (1978).

[6] V.V. Parkhomchuk, NIM A 441, p.9 (2000).

[7] S. Nagaitsev et al., PRL 96, 044801 (2006).

[8] Ya. Derbenev, "Electron cooling in solenoid with undulator", TJLAB Note 2001, unpublished.

[9] C. Nieter, J. Cary, J. Comp. Phys. 196 , p.448 (2004); http://www.txcorp.com.

[10] G. Bell et al.. "Numerical algorithms for modeling electron cooling in the presence of external fields", these proceedings.

[11] A. Sobol et al., "Quantifying reduction of the friction force due to magnet imperfections", these proceedings.

[12] M. Horndl et al., PRL 95, 243201 (2005).

[13] A. Wolf, H. Danared, I. Kaganovich (private communications, 2006).

[14] BETACOOL code, http://lepta.jinntru; A. Sidorin et al., NIM A 558, p.325 (2006). 